\title{
On-Surface Synthesis of a Nonplanar Porous Nanographene
}

Kun $\mathrm{Xu},{ }^{\dagger, \S_{(0)}}$ José I. Urgel, ${ }^{\dagger, \S_{(0)}}$ Kristjan Eimre, ${ }^{\ddagger}$ Marco Di Giovannantonio, ${ }^{\ddagger}$ Ashok Keerthi, ${ }^{\perp, \otimes}$

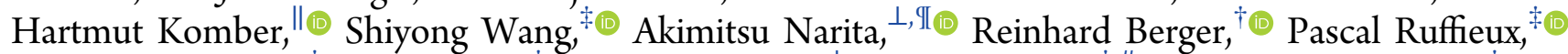
Carlo A. Pignedoli, ${ }^{\ddagger}$ Junzhi Liu, ${ }^{\dagger}$ Klaus Müllen, ${ }^{*, \perp}$ Roman Fasel, ${ }^{*},+,{ }^{\ddagger}$ and Xinliang Feng ${ }^{*},{ }^{\dagger}$

${ }^{\dagger}$ Center for Advancing Electronics Dresden, Department of Chemistry and Food Chemistry, Technische Universität Dresden, 01062 Dresden, Germany

${ }^{\sharp}$ Empa, Swiss Federal Laboratories for Materials Science and Technology, 8600 Dübendorf, Switzerland

${ }^{\perp}$ Max Planck Institute for Polymer Research, Ackermannweg 10, 55128 Mainz, Germany

${ }^{\otimes}$ National Graphene Institute, University of Manchester, Manchester M13 9PL, United Kingdom

"Leibniz-Institut für Polymerforschung Dresden e. V., Hohe Straße 6, 01069 Dresden, Germany

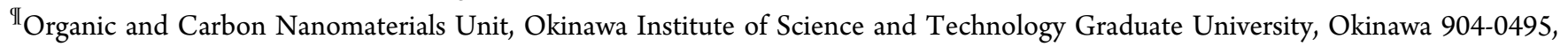
Japan

"Department of Chemistry and Biochemistry, University of Bern, 3012 Bern, Switzerland

Supporting Information

ABSTRACT: On-surface synthesis provides an effective approach toward the formation of graphene nanostructures that are difficult to achieve via traditional solution chemistry. Here, we report on the design and synthesis of a nonplanar porous nanographene with $78 s p^{2}$ carbon atoms, namely $\mathbf{C 7 8}$. Through a highly selective oxidative cyclodehydrogenation of 2,3,6,7,10,11-hexa(naphthalen-1yl)triphenylene (2), propeller nanographene precursor 1 was synthesized in solution. Interestingly, although 1 could not be cyclized further in solution, porous nanographene C78 was successfully achieved from 1 by on-surface assisted cyclodehydrogenation on $\mathrm{Au}(111)$. The structure and electronic properties of $\mathbf{C} 78$ have been investigated by means of scanning tunneling microscopy, noncontact atomic force microscopy, and scanning tunneling spectroscopy, complemented by computational investigations. Our results provide perspectives for the onsurface synthesis of porous graphene nanostructures, offering a promising strategy for the engineering of graphene materials with tailor-made properties.

G raphene nanostructures (also called "nanographenes") have gained increasing attention due to their great potential toward tailor-made organic materials with applications in electronics, ${ }^{1}$ optoelectronics, ${ }^{2}$ spintronics, ${ }^{3}$ chemical sensing, ${ }^{4}$ gas storage, ${ }^{5}$ etc. The synthesis of graphene nanostructures via top-down methods such as exfoliation ${ }^{6 a, b}$ presents well-known disadvantages such as nonregular edge structures or uncontrollable sizes. In contrast, the bottom-up organic synthesis approach has emerged in the last decades as a powerful tool to design structurally well-defined nanographenes. $^{6 c-e}$ The properties of nanographenes are largely determined by their structural characteristics including their size and edge structure. The controlled introduction of lattice defects/nanopores in the basal plane of the $s p^{2}$ carbon skeleton represents an alternative strategy to influence the properties of such nanographenes. ${ }^{7}$ Depending on their internal substructure, nanopores can be planar or nonplanar. ${ }^{8}$ Typically, planar nanopores 9 exhibit internal zigzag and armchair like substructures (Figure 1, red, A-D). Differently, nonplanar

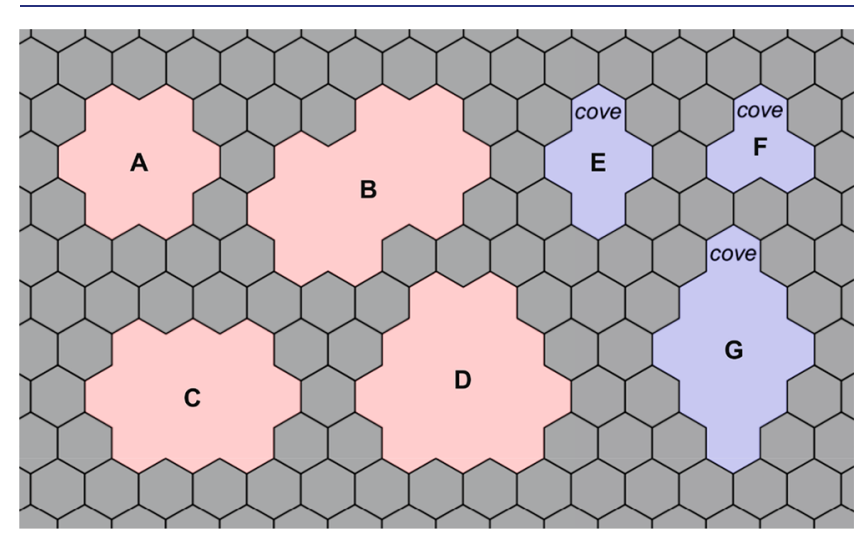

Figure 1. Examples of planar (red, A-D) and nonplanar (blue, E-G) nanopores in graphene.

nanopores contain the so-called cove-type subunits (Figure 1 , blue, $E-G)$. In addition, the presence of these nanopores in graphene nanostructures facilitates the access to fascinating properties with potential applications in water desalination, sensing, and gas separation. ${ }^{4,5,10}$

Despite the progress made in recent years, the bottom-up synthesis of graphene nanostructures ${ }^{6 c-e}$ with well-defined nanopores is a remaining challenge. ${ }^{9,11,12}$ Specifically, large precursors of polyphenylenes may present a mismatching of topology which leads to partial cyclodehydrogenation. ${ }^{13}$ Recently, we reported the introduction of a planar nanopore (type A in Figure 1) into a graphene nanostructure, C216, via solution-based cyclodehydrogenation of a polyphenylene

Received: April 2, 2019

Published: May 2, 2019 
precursor with preinstalled porous macrocycle, which leads to an increased HOMO-LUMO gap compared to that of its parent nanographene C222. ${ }^{11}$ Although a few graphene nanostructures containing planar nanopores have been achieved through on-surface ${ }^{14}$ synthesis, ${ }^{9}$ the access to nanographenes containing nonplanar nanopores is still missing.

Herein, we introduce an exemplary approach toward the design and on-surface formation of a nonplanar, porous nanographene, namely the $\mathbf{C 7 8}$, studied on a coinage metal surface under ultrahigh vacuum (UHV) conditions (Scheme 1). Compound $\mathbf{C 7 8}$ can be considered as a derivative of $\mathbf{C 8 4}$,

Scheme 1. Concept, Design, and Synthesis of the Nonplanar, Porous Nanographene C78 via On-Surface Synthesis*

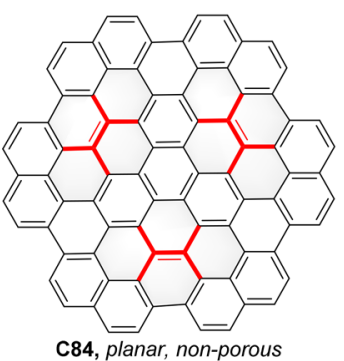

$-3 C=C$

C84, planar, non-porous

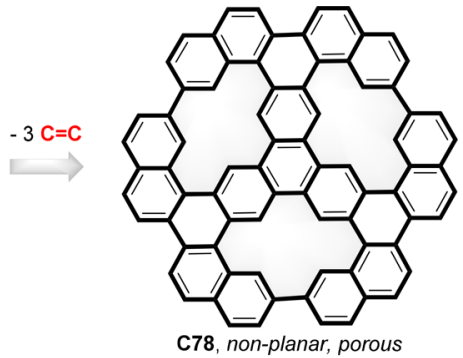

c78, non-planar, porous (b)

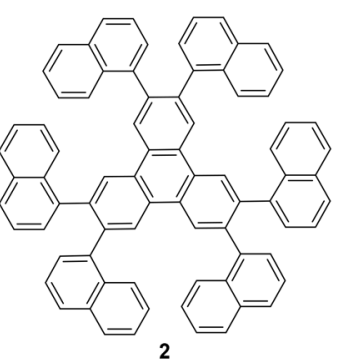

(a)

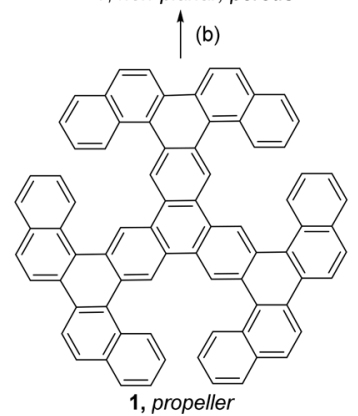

*(a) DDQ (2,3-dichloro-5,6-dicyano-1,4-benzoquinone, 3.6 equiv), $\mathrm{CH}_{2} \mathrm{Cl}_{2} / \mathrm{CF}_{3} \mathrm{SO}_{3} \mathrm{H}(9 / 1), 0{ }^{\circ} \mathrm{C}$ to room temperature, $15 \mathrm{~h}, 75 \%$. (b) On $\mathrm{Au}(111), 350{ }^{\circ} \mathrm{C}$.

which has a planar configuration with $84 s p^{2}$ carbon atoms, by removing three double bonds from its carbon framework and forming three nanopores with the topology of the rim of a pyrene molecule (type $\mathrm{E}$ in Figure 1 and Scheme 1). The target molecule C78 was successfully achieved via surfaceassisted cyclodehydrogenation of the propeller precursor 1, which was synthesized via regioselective cyclodehydrogenation of 2,3,6,7,10,11-hexa(naphthalen-1-yl)triphenylene (2) in solution. The structure of $\mathbf{C 7 8}$ is evidenced by scanning tunneling microscopy (STM), and complemented by densityfunctional theory (DFT) calculations. The chemical structure of the final porous nanographene is determined by noncontact atomic force microscopy (nc-AFM), and its electronic properties are studied by scanning tunneling spectroscopy (STS), which indicates an increased bandgap from $2.4 \mathrm{eV}$ for the planar C84 (obtained by GW calculations) to $3.0 \mathrm{eV}$ for the nonplanar porous $\mathbf{C 7 8}$ on $\mathrm{Au}(111)$.

Compound $\mathbf{2}$ was synthesized in solution via 6-fold Suzuki coupling with a $96 \%$ yield (Scheme S1, Table S1, and Figures $\mathrm{S} 1-\mathrm{S} 8$ in the Supporting Information). Our preliminary attempts to use $\mathbf{2}$ directly for the on-surface formation of C78 via cyclodehydrogenation were not successful. Specifically, the sublimation of a submonolayer coverage of $\mathbf{2}$ onto the
$\mathrm{Au}(111)$ substrate held at room temperature and subsequently annealed to $250{ }^{\circ} \mathrm{C}$ to induce cyclodehydrogenation gave mostly rise to the unexpected formation of individual illdefined nanostructures which coexist with $\sim 5 \%$ of triblade-like propeller molecules (Figure S9). To the best of our knowledge, the formation of pentalene units observed in the triblade-like moleculehas not been reported in on-surface synthesis, and it may offer a new way to access pentalene containing nanographenes. The flexibility and high reactivity of the naphthyl groups in compound 2, as well as the steric congestion of the target $\mathbf{C 7 8}$ molecule may account for this unexpected result.

To overcome the limitations observed in the on-surface formation of $\mathbf{C 7 8}$ starting from 2, we designed precursor 1, which has a more rigid preplanarized structure and less reactive sites. The synthesis of $\mathbf{1}$ was carried out by oxidation of $\mathbf{2}$ using 2,3-dichloro-5,6-dicyano-1,4-benzoquinone (DDQ) in a mixture of dichloromethane and triflic acid at $0{ }^{\circ} \mathrm{C}$ in $75 \%$ yield (Scheme S2 and Figures S10-S14). Our attempts of solution cyclodehydrogenation toward C78 from 1 was unfortunately unsuccessful. The propeller structure of $\mathbf{1}$, which pushes the $\mathrm{C}-\mathrm{H}$ bonds in the rims far away from each other, may account for the challenge of the solution process.

After sublimation of compound 1 under UHV conditions onto an atomically clean $\mathrm{Au}(111)$ surface held at room temperature, large-scale STM images (Figure $2 \mathrm{a}$ ) reveal the
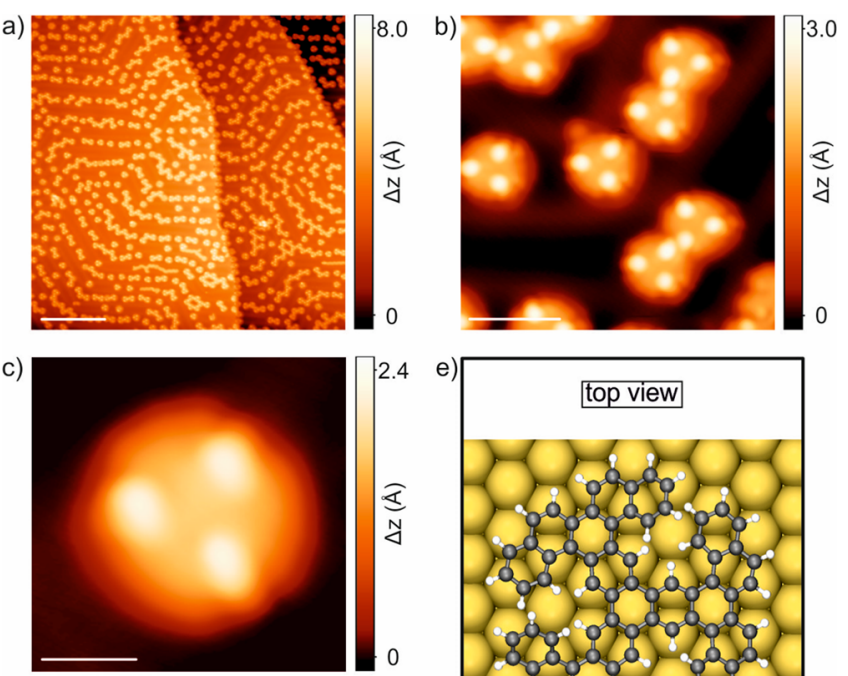

d)
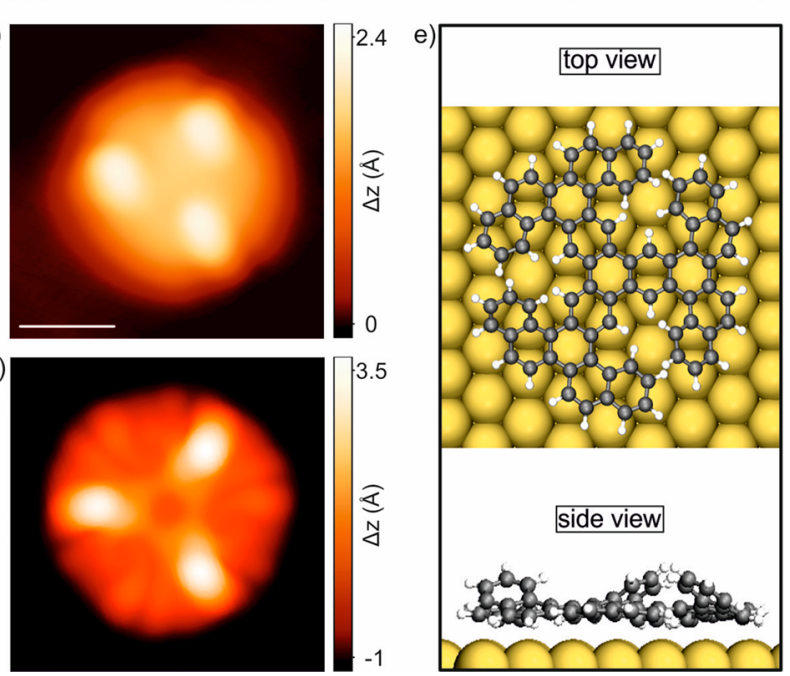

Figure 2. Identification of $\mathbf{1}$ after deposition on $\operatorname{Au}(111)$. (a,b) Overview STM topography images of the surface after room temperature deposition of $1 . V_{\mathrm{b}}=-1.0 \mathrm{~V}$ and $-2 \mathrm{mV}, I_{\mathrm{t}}=100 \mathrm{pA}$, scale bar $=20$ and $3 \mathrm{~nm}$, respectively. (c) Magnified view of one of the rounded species observed in panel $(a, b)$ evidencing the presence of three bright peripheral lobes per molecule. $V_{\mathrm{b}}=-0.1 \mathrm{~V}, I_{\mathrm{t}}=25 \mathrm{pA}$, scale bar $=1 \mathrm{~nm}$. (d) DFT-simulated STM image of the molecule highlighted in panel c. (e) Top (upper panel) and side (lower panel) views of the DFT equilibrium geometry of 1 on $\mathrm{Au}(111)$. 
presence of several self-assembled chains that follow the $\mathrm{Au}(111)$ herringbone reconstruction, along with sporadically distributed individual molecules. Figure $2 \mathrm{~b}$ shows a highresolution STM image where rounded molecular species, which present three bright peripheral lobes with a maximum apparent height of $2.5 \AA$ (measured at a sample bias of $-1 \mathrm{~V}$ ), interact between them due to the nonplanar conformation adopted by the molecules on the surface. Additionally, highresolution STM images acquired with a CO-functionalized tip allow us to discern $\mathrm{R}$ and $\mathrm{S}$ enantiomers within the same image (Figure S15). The experimental features of an individual molecule observed in Figure $2 \mathrm{c}$ are well reproduced by the DFT-optimized geometry and the corresponding STM simulation of 1 on the $\mathrm{Au}(111)$ surface (Figure 2d,e), which shows that 1 adopts a consecutive "up and down" configuration (with an adsorption height of $3.4 \AA$ with respect to the underlying surface), indicating a typical propeller-type conformation on the surface. ${ }^{15}$

Annealing the substrate at $180{ }^{\circ} \mathrm{C}$ does not give rise to significant modifications in the molecular structure of $\mathbf{1}$ and just a small number of molecules $(\sim 10 \%)$ present a different appearance (Figure S16). Only after a second annealing step to $350{ }^{\circ} \mathrm{C}$, the subsequent formation of the targeted porous $\mathbf{C 7 8}$ molecule is achieved by the cyclodehydrogenation of $\mathbf{1}$. Figure 3a,b depicts the high-resolution STM images where the molecular species appear as circularly shaped molecules without any prominent lobes which is attributed to the formation of C78 (63\% out of 150 molecules). It should be noted that some of the molecules remained with one protrusion, implying an incomplete cyclodehydrogenation of the molecular precursor.

To confirm the chemical structure of $\mathbf{C 7 8}$, noncontact atomic force microscopy (nc-AFM) measurements using a CO-functionalized tip were performed. ${ }^{16}$ Figure $3 \mathrm{c}$ depicts the resulting constant-height frequency-shift image. Herein, the nonplanarity which arises due to the intramolecular $\mathrm{H}-\mathrm{H}$ steric hindrance is clearly manifested. The periphery of the molecular backbone shows three features of increased frequency shift while the center of the molecule is closer to the gold surface and therefore no obvious features could be discerned in the nc-AFM image. In addition, the simulated ncAFM image depicted in Figure $3 \mathrm{~d}$ yields excellent correspondence to the experimental image. This nonplanarity is well visible in the DFT-optimized structure (Figure 3e) of C78 on $\mathrm{Au}(111)$, which shows how the new $\mathrm{C}-\mathrm{C}$ bonds formed after cyclodehydrogenation are slightly bent out-ofplane (a dihedral angle of $4.4^{\circ}$, with an adsorption height of 3.1 $\AA$ with respect to the underlying surface). Altogether, this provides conclusive evidence for the structural assignment of the porous $\mathbf{C 7 8}$ molecule on the $\mathrm{Au}(111)$ surface.

Next, we performed STS measurements on $\mathbf{C 7 8}$ to probe its electronic structure. Voltage-dependent differential conductance spectra $(\mathrm{d} I / \mathrm{d} V$ vs $V)$ display peaks in the density of states at -1.2 and $+1.8 \mathrm{~V}$ (Figure $4 \mathrm{a}$ ), which are attributed to the positive and negative ion resonances (PIR and NIR, respectively) deriving from the highest occupied and the lowest unoccupied molecular orbitals (HOMO and LUMO) of C78. Therefore, the measured gap of this nonplanar porous nanographene is $3.0 \mathrm{eV}$ on $\mathrm{Au}(111)$, which is in good agreement with the value of $3.1 \mathrm{eV}$ obtained by applying image charge corrections to the eigenvalue self-consistent $G W$ gap calculated for the isolated molecule adsorbed on $\mathrm{Au}(111)$. This value contrasts with the gap of $2.4 \mathrm{eV}$ calculated for the
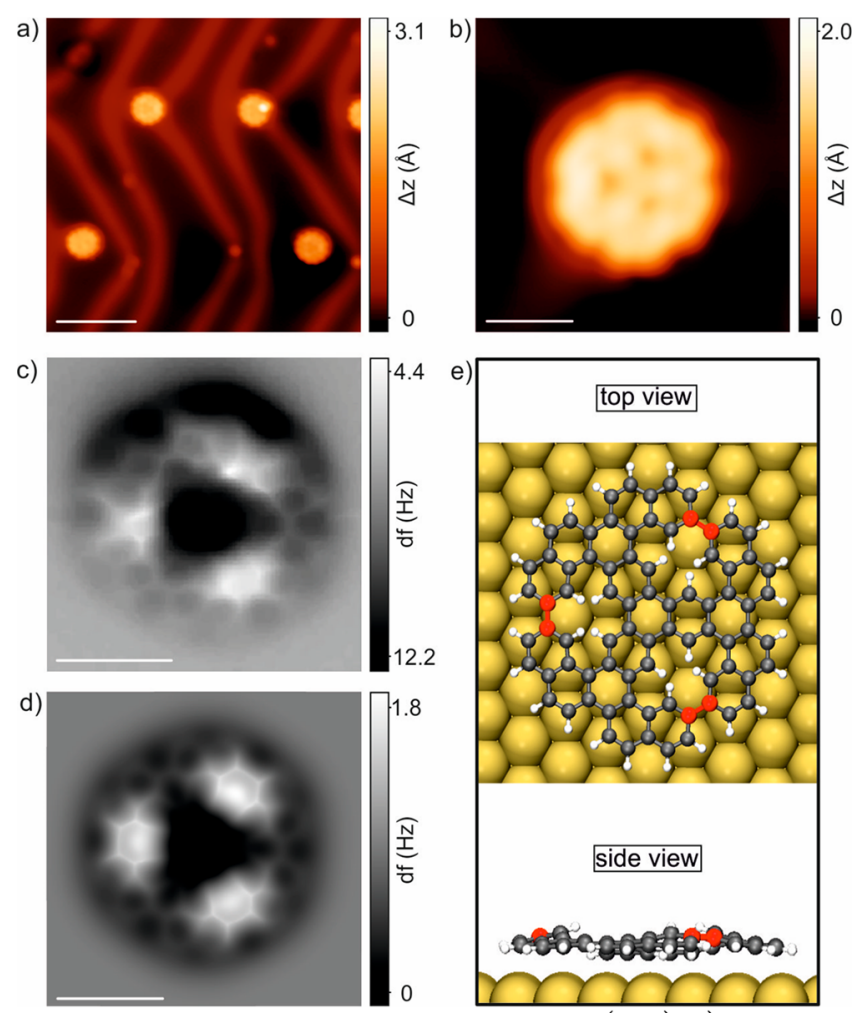

Figure 3. On-surface synthesis of C78 on $\mathrm{Au}(111)$. (a) Overview STM topography image of the surface after annealing at $350{ }^{\circ} \mathrm{C}$, showing the predominant presence of $\mathbf{C 7 8}$ molecules. $V_{\mathrm{b}}=-1.2 \mathrm{~V}, \mathrm{I}_{\mathrm{t}}$ $=175 \mathrm{pA}$, scale bar $=4 \mathrm{~nm}$. (b) High-resolution STM image of an isolated molecule. $V_{\mathrm{b}}=0.1 \mathrm{~V}, I_{\mathrm{t}}=200 \mathrm{pA}$, scale bar $=1 \mathrm{~nm}$. (c) Constant-height frequency-shift nc-AFM image of panel $b$ acquired with a CO-functionalized tip ( $\mathrm{z}$ offset $-35 \mathrm{pm}$ below STM set point: $5 \mathrm{mV}, 10 \mathrm{pA}$ ). Scale bar $=1 \mathrm{~nm}$. (d) Simulated nc-AFM image of panel c. (e) Top and side views of the DFT equilibrium geometry of $\mathrm{C} 78$ on $\mathrm{Au}(111)$. Bonds and atoms highlighted in red show an out-ofplane bending contributing to the distinguishing features in the ncAFM images.

planar, nonporous C84, which isdue to the extended $\pi$-system being disrupted by the pores and by the nonplanarity of the molecule.

To investigate the impact of nonplanar nanopores such as those observed for $\mathbf{C 7 8}$ on the electronic properties of extended 2D graphene, we performed tight-binding and DFT band structure calculations. The results show that such pores introduce flat bands and open a band in pristine graphene (Figure S17). The band gap opening is analogous to the increase of the HOMO-LUMO gap of $\mathbf{C 7 8}$ with respect to C84. Constant-height maps of the $\mathrm{d} I / \mathrm{d} V$ signal of a $\mathbf{C 7 8}$ on $\mathrm{Au}(111)$ acquired with a CO-functionalized tip at the energetic positions of the PIR (top) and the NIR (bottom), together with the corresponding STM topography images are reported in Figure 4b (see Figure S18 for comparison with the constantcurrent $\mathrm{d} I / \mathrm{d} V$ maps of $\mathbf{C 7 8})$. In addition, the DFT-calculated local density of states (LDOS) maps of the HOMO and the LUMO of C78 (Figure 4b, right), evaluated at a height of $3 \AA$ above the molecular plane, indicate a notable agreement with the experimental $\mathrm{d} I / \mathrm{d} V$ maps, which allows us to conclude that the conductance features observed in the $\mathrm{d} I / \mathrm{d} V$ maps can be correlated with the spatial distribution of the probability densities of the corresponding frontier orbital wave functions. 
a)

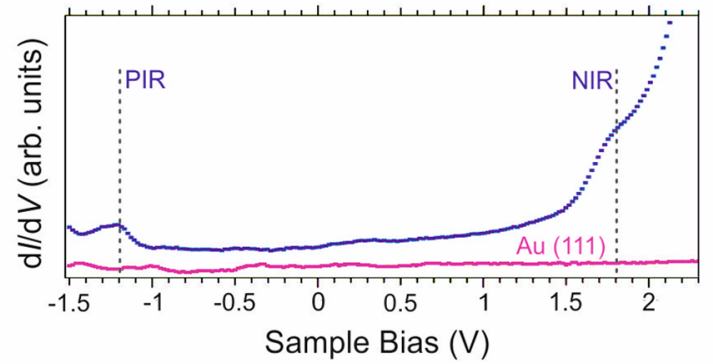

b) Topography
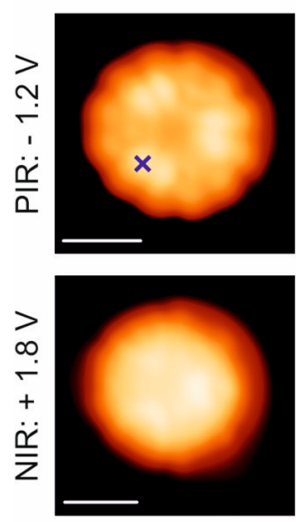

Low
Exp. $d / / d V$
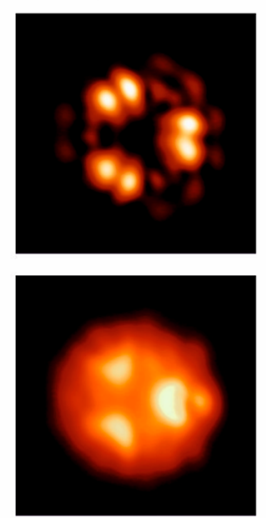

Low
Calc. $d / / d V$
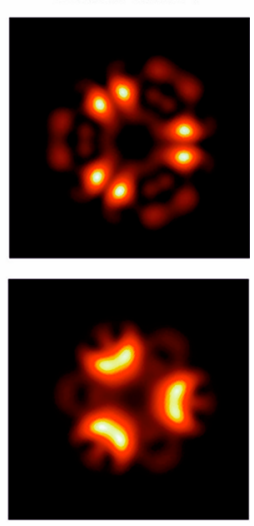

High
Figure 4. Electronic properties of $\mathbf{C 7 8}$. (a) $\mathrm{d} I / \mathrm{d} V$ spectrum acquired on the molecule at the position indicated by the blue cross in (b) (blue line), revealing a band gap of $3.0 \mathrm{eV}$, and reference spectrum taken on the bare $\mathrm{Au}(111)$ surface (pink line). (b) High-resolution STM images (left), simultaneously acquired constant-height differential conductance $(\mathrm{d} I / \mathrm{d} V)$ maps (center) and corresponding DFTcalculated LDOS maps (right) at the energetic positions corresponding to the PIR (top) and the NIR (bottom). Tunneling parameters for the STM images and the associated $\mathrm{d} I / \mathrm{d} V$ maps: PIR $\left(V_{\mathrm{b}}=-1.2 \mathrm{~V}, I_{\mathrm{t}}\right.$ $=175 \mathrm{pA}) ; \operatorname{NIR}\left(V_{\mathrm{b}}=1.8 \mathrm{~V}, I_{\mathrm{t}}=175 \mathrm{pA}\right)$. All scale bars $=1 \mathrm{~nm}$.

In conclusion, we demonstrated a synthetic pathway for the on-surface synthesis of a well-defined triple-porous nanographene on $\mathrm{Au}(111)$. Based on the parent propeller precursor 1, the nonplanar, porous $\mathbf{C 7 8}$ molecule has been achieved via on-surface cyclodehydrogenation and its structure has been clearly elucidated by STM and nc-AFM. STS studies together with theoretical calculations revealed that the $\mathbf{C 7 8}$ molecule exhibits an experimental electronic gap of $3.0 \mathrm{eV}(3.1 \mathrm{eV}$ obtained by GW calculations), which differs from the $2.4 \mathrm{eV}$ calculated for the planar, nonporous C84. This porous nanographene can serve as a model structure and help to understand the effect of nonplanar nanopores in graphene nanostructures. Our future work will focus on the synthesis of nanographenes with various nanopores and larger $\pi$-systems, as well as porous graphene nanoribbons, where the dependence of the intrinsic electronic properties on the topology and the symmetry of atomic sites allow for the engineering of novel functionalities.

\section{ASSOCIATED CONTENT}

\section{S Supporting Information}

The Supporting Information is available free of charge on the ACS Publications website at DOI: 10.1021/jacs.9b03554.
Experimental details, synthesis, characterizations, computational studies, and NMR spectra (PDF)

Data for $\mathrm{C}_{78} \mathrm{H}_{48}$ (CIF)

\section{AUTHOR INFORMATION}

\section{Corresponding Authors}

*xinliang.feng@tu-dresden.de

*roman.fasel@empa.ch

*muellen@mpip-mainz.mpg.de

ORCID $\odot$

Kun Xu: 0000-0002-4516-7563

José I. Urgel: 0000-0001-7608-2979

Ashok Keerthi: 0000-0002-8479-4762

Hartmut Komber: 0000-0001-6176-6737

Shiyong Wang: 0000-0001-6603-9926

Akimitsu Narita: 0000-0002-3625-522X

Reinhard Berger: 0000-0002-8959-7821

Pascal Ruffieux: 0000-0001-5729-5354

Carlo A. Pignedoli: 0000-0002-8273-6390

Junzhi Liu: 0000-0001-7146-0942

Roman Fasel: 0000-0002-1553-6487

Xinliang Feng: 0000-0003-3885-2703

\section{Author Contributions}

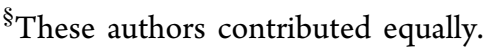

\section{Notes}

The authors declare no competing financial interest.

\section{ACKNOWLEDGMENTS}

This work was supported by the Swiss National Science Foundation, the Office of Naval Research BRC program, the European Union's Horizon 2020 research, and innovation program under grant agreement no. 696656 (Graphene Flagship Core2), ERC Consolidator Grant (T2DCP, no. 819698), the German Research Foundation (DFG) within the Cluster of Excellence "Center for Advancing Electronics Dresden (cfaed)" and EnhanceNano (no. 391979941) as well as the European Social Fund and the Federal State of Saxony (ESF-Project "GRAPHD”, TU Dresden) and a grant from the Swiss National Supercomputing Centre (CSCS) under project ID s746 and s904. The Swiss National Centre for Computational Design and Discovery of Novel Materials (MARVEL) is acknowledged for financial support. We acknowledge Dr. Dieter Schollmeyer at Johannes GutenbergUniversity for crystal structure analysis.

\section{REFERENCES}

(1) (a) Wu, J.; Pisula, W.; Müllen, K. Graphenes as Potential Material for Electronics. Chem. Rev. 2007, 107, 718-747. (b) Jiang, W.; Li, Y.; Wang, Z. Tailor-Made Rylene Arrays for High Performance n-Channel Semiconductors. Acc. Chem. Res. 2014, 47, 3135-3147.

(2) Bonaccorso, F.; Hasan, S. T.; Ferrari, A. C. Graphene Photonics and Optoelectronics. Nat. Photonics 2010, 4, 611-622.

(3) Han, W.; Kawakami, R. K.; Gmitra, M.; Fabian, J. Graphene Spintronics. Nat. Nanotechnol. 2014, 9, 794-807.

(4) Yuan, W.; Chen, J.; Shi, G. Nanoporous Graphene Material. Mater. Today 2014, 17, 77-85.

(5) Gadipelli, S.; Guo, Z. X. Graphene-based Materials: Synthesis and Gas Sorption, Storage and Separation. Prog. Mater. Sci. 2015, 69, $1-60$.

(6) (a) Novoselov, K. S.; Geim, A. K.; Morozov, S. V.; Jiang, D.; Zhang, Y.; Dubonos, S. V.; Grigorieva, I. V.; Firsov, A. A. Electric Field Effect in Atomically Thin Carbon Films. Science 2004, 306, 
666-669. (b) Li, X.; Wang, X.; Zhang, L.; Lee, S.; Dai, H. Chemically Derived, Ultrasmooth Graphene Nanoribbon Semiconductors. Science 2008, 319, 1229-1232. (c) Chen, L.; Hernandez, Y.; Feng, X.; Müllen, K. From Nanographene and Graphene Nanoribbons to Graphene Sheets: Chemical Synthesis. Angew. Chem., Int. Ed. 2012, 51, 7640-7654. (d) Kawasumi, K.; Zhang, Q.; Segawa, Y.; Scott, L. T.; Itami, K. A Grossly Warped Nanographene and the Consequences of Multiple Odd-membered-ring Defects. Nat. Chem. 2013, 5, 739744. (e) Pun, S. H.; Miao, Q. Toward Negatively Curved Carbons. Acc. Chem. Res. 2018, 51, 1630-1642.

(7) (a) Chen, J.; Li, L.; Cullen, W. G.; Williams, E. D.; Fuhrer, M. S. Tunable Kondo effect in graphene with defects. Nat. Phys. 2011, 7, 535-538. (b) Lahiri, J.; Lin, Y.; Bozkurt, P.; Oleynik, I. I.; Batzill, M. An Extended Defect in Graphene as a Metallic Wire. Nat. Nanotechnol. 2010, 5, 326-329.

(8) Govind Rajan, A.; Silmore, K. S.; Swett, J.; Robertson, A. W.; Warner, J. H.; Blankschtein, D.; Strano, M. S. Addressing the Isomer Cataloguing Problem for Nanopores in Two-dimensional Materials. Nat. Mater. 2019, 18, 129-135.

(9) (a) Bieri, M.; Nguyen, M.; Gröning, O.; Cai, J.; Treier, M.; AïtMansour, K.; Ruffieux, P.; Pignedoli, C. A.; Passerone, D.; Kastler, M.; Müllen, K.; Fasel, R. Two-Dimensional Polymer Formation on Surfaces: Insight into the Roles of Precursor Mobility and Reactivity. J. Am. Chem. Soc. 2010, 132, 16669-16676. (b) Ammon, M.; Sander, T.; Maier, S. On-Surface Synthesis of Porous Carbon Nanoribbons from Polymer Chains. J. Am. Chem. Soc. 2017, 139, 12976-12984. (c) Moreno, C.; Vilas-Varela, M.; Kretz, B.; Garcia-Lekue, A.; Costache, M. V.; Paradinas, M.; Panighel, M.; Ceballos, G.; Valenzuela, S. O.; Peña, D.; Mugarza, A. Bottom-up Synthesis of Multifunctional Nanoporous Graphene. Science 2018, 360, 199-203.

(10) (a) Venkatesan, B. M.; Bashir, R. Nanopore Sensors for Nucleic Acid Analysis. Nat. Nanotechnol. 2011, 6, 615-624. (b) Surwade, S. P.; Smirnov, S. N.; Vlassiouk, I. V.; Unocic, R. R.; Veith, G. M.; Dai, S.; Mahurin, S. M. Water Desalination Using Nanoporous Single-layer Graphene. Nat. Nanotechnol. 2015, 10, 459-464.

(11) Beser, U.; Kastler, M.; Maghsoumi, A.; Wagner, M.; Castiglioni, C.; Tommasini, M.; Narita, A.; Feng, X.; Müllen, K. A C216Nanographene Molecule with Defined Cavity as Extended Coronoid. J. Am. Chem. Soc. 2016, 138, 4322-4325.

(12) Elegant examples of phenylene-based porous cabon nanostructures: (a) Ikemoto, K.; Kobayashi, R.; Sato, S.; Isobe, H. Synthesis and Bowl-in-Bowl Assembly of a Geodesic Phenylene Bowl. Angew. Chem. Angew. Chem., Int. Ed. 2017, 56, 6511-6514. (b) Sun, Z.; Ikemoto, K.; Fukunaga, T. M.; Koretsune, T.; Arita, R.; Sato, S.; Isobe, H. Finite Phenine Nanotubes with Periodic Vacancy Defects.

Science 2019, 363, 151-155.

(13) Narita, A. Synthesis of Structurally Defined Nanographene Materials through Oxidative Cyclodehydrogenation. Synthetic Methods for Conjugated Polymer and Carbon Materials. In Synthetic Methods for Conjugated Polymers and Carbon Materials; Leclerc, M., Morin, J., Eds.; Wiley-VCH Verlag GmbH \& Co. KGaA: Weinheim, Germany, 2017; pp 183-228.

(14) (a) Shen, Q.; Gao, H.; Fuchs, H. Frontiers of On-surface Synthesis: From Principles to Applications. Nano Today 2017, 13, 77-96. (b) Rogers, C.; Chen, C.; Pedramrazi, Z.; Omrani, A. A.; Tsai, H.; Jung, H. S.; Lin, S.; Crommie, M. F.; Fischer, F. R. Closing the Nanographene Gap: Surface-Assisted Synthesis of Peripentacene from 6,6'-Bipentacene Precursors. Angew. Chem., Int. Ed. 2015, 54, 1514315146.

(15) (a) Ernst, K. Stereochemical Recognition of Helicenes on Metal Surfaces. Acc. Chem. Res. 2016, 49, 1182-1190.

(16) (a) Gross, L.; Mohn, F.; Moll, N.; Liljeroth, P.; Meyer, G. The Chemical Structure of a Molecule Resolved by Atomic Force Microscopy. Science 2009, 325, 1110-1114. (b) Gross, L.; Mohn, F.; Moll, N.; Schuler, B.; Criado, A.; Guitián, E.; Peña, D.; Gourdon, A.; Meyer, G. Bond-Order Discrimination by Atomic Force Microscopy. Science 2012, 337, 1326-1329. 